INPLASY

PROTOCOL

To cite: Machado et al. School

Sport, conceptual and operational realities: A

Systematic Review. Inplasy protocol 2021100027. doi:

10.37766/inplasy2021.10.0027

Received: 08 October 2021

Published: 08 October 2021

Corresponding author:

Rui Machado

rmachado@ipmaia.pt

Author Affiliation:

Investigation Group of Coruña

University dedicated to

Education, Health and Physical

Activity: Gender Studies - A

Coruña, Galicia, Spain.

Support: Nothing to declare.

Review Stage at time of this submission: Preliminary

searches.

Conflicts of interest:

None declared.

\section{School Sport, conceptual and operational realities: A Systematic Review}

Machado, R1; Sarmento, H2; Valeiro, M33; Tobio, G4; Duarte, D5; Teques, $\mathrm{P}^{6}$.

Review question / Objective: What is the concept of School Sport in different countries and how they operationalize it? Condition being studied: The School Sports in several countries, are a crucial element of the educational system and assumes an increseally importance for the physical activity and health programs. In this pandemic period, the physical activity and the wellbeing of children and young adults have come to the center of a large discussion. The school sports, in this context, are assumed as player in consideration, but as countries discuss it, are noted different understandings of the school sports concept in each country as well different approaches and applications of it.

INPLASY registration number: This protocol was registered with the International Platform of Registered Systematic Review and Meta-Analysis Protocols (INPLASY) on 08 October 2021 and was last updated on 08 October 2021 (registration number INPLASY2021100027).

\section{INTRODUCTION}

Review question / Objective: What is the concept of School Sport in different countries and how they operationalize it?

Condition being studied: The School Sports in several countries, are a crucial element of the educational system and assumes an increseally importance for the physical activity and health programs. In this pandemic period, the physical activity and the wellbeing of children and young adults have come to the center of a large discussion. The school sports, in this context, are assumed as player in consideration, but as countries discuss it, are noted different understandings of the 
school sports concept in each country as well different approaches and applications of it.

\section{METHODS}

Search strategy: "school* sport"” OR "sport" school*" OR "after-school sport"” OR "sport* in school* age*" OR "extracurricular sport"”.

Participant or population: School sports problematic.

Intervention: The School Sports are worldwide spread, but as the discussion involves different countries, existing concept and operationalization problems turn deeper insight quite difficult. Aware of this, we argue that in the first hand is necessary to clarify these two points and enable further and deeper interventions.

\section{Comparator: Not Applicable.}

Study designs to be included: Five online databases (sources) will be searched for literature: Web of Science; Sport Discus; Psyclnfo, PubMed and ERIC. Additionally, we will conduct a search to identify relevant documents that are publish by relevant organisations: OECD (Organisation for Economic Co-operation and Development), UNESCO (United Nations Educational, Scientific and Cultural Organization), European Union and WHO (World Health Organisation).

Eligibility criteria: The search period select will be since 2010, because school sports policies have changed significantly in the last decade and we aim to be focused on actual data.To ensure the quality of the search, we will limit it to original articles, writing in English Language. On the other hand, we will not include books, chapters, conferences papers or any document that do not focuses in School Sports.

Information sources: Five online databases (sources) will be searched for literature: Web of Science; Sport Discus; Psyclnfo, PubMed and ERIC. Additionally, we will conduct a search to identify relevant documents that are publish by relevant organisations: OECD (Organisation for Economic Co-operation and Development), UNESCO (United Nations Educational, Scientific and Cultural Organization), European Union and WHO (World Health Organisation). The search period select will be since 2010, because school sports policies have changed significantly in the last decade and we aim to be focused on actual data. To ensure the quality of the search, we will limit it to original articles, writing in English Language. On the other hand, we will not include books, chapters, conferences papers or any document that do not focuses in School Sports.

Main outcome(s): The two main outcomes of this review are the: (1) Concept (is under which public department umbrella? what are the main objectives?; how it is financed? ...), and; (2) how each Country operationalizes the School Sports (who is accountable? is a mandatory offer for pupils?; is for all ages?, ...).

\section{Additional outcome(s): None.}

Quality assessment / Risk of bias analysis: The third and final step in the data handling process is a quality control of the included studies using a combination of different quality assessment tools for quantitative/ qualitative research.

Strategy of data synthesis: As a first step in the data handling process, titles and abstracts of all papers retrieved by the search strategy will be screened for relevance and all those that are clearly irrelevant will be discarded. If the result is not clearly irrelevant, the full text will be downloaded. As a second step, two review team members will independently assess the eligibility of the studies by using the predefined inclusion and exclusion criteria. Any disagreements on whether or not to include a specific study will be resolved by discussion between the reviewers.

Subgroup analysis: None Planned.

Sensitivity analysis: None. 
Language: English.

Country(ies) involved: Portugal and Spain.

Keywords: School Sport; Extracurricular Activities; Concept.

Dissemination plans: A paper will be submitted to one of the leading journals of Physical Education and Sports.

Contributions of each author:

Author 1 - Rui Menezes Machado - The Author 1 is the responsible for the research plan and its execution.

Email: rmachado@ipmaia.pt

Author 2 - Hugo Sarmento - The Author 2 is the supervisor and responsible for the quality assessment and risk of bias analysis.

Email: hugo.sarmento@uc.pt

Author 3 - Miguel Valeiro - The Author 3 will contributed to the development of the selection criteria, and the risk of bias assessment strategy.

Email: miguel.gonzalez.valeiro@udc.es

Author 4 - Gabriel Torres Tobio - The Author 4 read, provided feedback and approved the final manuscript.

Email: gabriel.torres@udc.es

Author 5 - Daniel Duarte - The Author 5 read, provided feedback and approved the final manuscript.

Email: dfduarte@ipmaia.pt

Author 6 - Pedro Teques - The Author 6 read, provided feedback and approved the final manuscript.

Email: pteques@ipmaia.pt 